Trauma Berufskrankh 2008 · 10[Suppl 1]:95-98 DOI 10.1007/s10039-007-1249-9

Online publiziert: 15. Dezemberg 2007

๑) Springer Medizin Verlag 2007

\author{
D. Haack \\ Berufsverband Niedergelassener Chirurgen, Hamburg
}

\section{Kommt der} Durchgangsarzt auf
seine Kosten?
Die gestellte Frage, ob der Durchgangsarzt auf seine Kosten kommt, ist mit einem „nein“" zu beantworten. Dies soll nicht unbegründet bleiben.

\section{Derzeit gültige Gebührenordnung}

Die Durchgangsärzte arbeiten mit einer UV-GOÄ, auf die Folgendes zutrifft:

- Sie entspricht der Systematik der GOÄ von 1996 .

- Es wurden die damaligen Leistungslegenden und Punktzahlen übernommen.

- Eine betriebswirtschaftliche Kalkulation wie im EBM 2000 plus existiert in der UV-GOÄ nicht.

Die Berufsgenossenschaften haben im Januar 2005 eine Änderung der ambulanten operativen Abrechnung vorgenommen. Nachdem jahrelang keine Zuschläge bei ambulanten Operationen abgerechnet werden konnten, gibt es diese jetzt für die Durchführung ambulanter Operationen und für die ambulanten Anästhesieleistungen.

Die Kosten gliedern sich in der UVGOÄ auf in:

- Ärztliche Gebühren,

- Entschädigung und

- Ersatz von Auslagen (Ausnahmen!).

Im Rahmen der ärztlichen Gebühren abgegolten sind die Praxiskosten - der Begriff Operationssaalbenutzung existiert nicht - die Kosten für Sprechstundenbedarf und die für Instrumente und Apparate.

Die niedergelassenen Ärzte können aufgrund von Verhandlungen der DKG mit den UV-Trägern als besondere Kosten ihre Sachkosten mit Pauschalen abrechnen. Bei diesen Verhandlungen waren jedoch die KBV, die KV und die BV nicht beteiligt! Zudem wurden die vereinbarten Pauschalen seit dem 01.03.2007 trotz MWST-Erhöhung! - teilweise massiv gesenkt. In diesem Kontext ist sicherlich gut nachvollziehbar, dass Krankenhäuser ihre Einkäufe unter ganz anderen Bedingungen und auch in großen Mengen tätigen können als niedergelassene Chirurgen. Ich empfehle daher, die angefallenen Sachkosten bei der Rechnungserstellung individuell zu hinterlegen.

\section{Kosten}

Die anfallenden Kosten für Materialien können z. T. erheblich sein, wie aus - Tab. 1 und 2 hervorgeht. In Letzterer wurden beispielhaft die Einkaufspreise für die Sachkosten bei einem Kleinsteingriff im OP unseres Operationszentrums aufgelistet. Nicht berücksichtigt dabei sind die Kosten für

- Aufbereitung der Instrumente

- Sterilisation der Instrumente

- Ersatz bzw. Reparatur der Geräte und Instrumente

- Vor- und Nachbereitung des OP

- Unterhalt wie Strom, Miete, Praxiskosten

- Kosten für Operationsschwester, Springer, Putzfrau, Anästhesieschwester

Diese Kosten existieren auch in den Berechnungen der BG nicht!

Die Operationssaalbenutzung wird im Operationszentrum des Autors, das be- triebswirtschaftlich dem Mittelwert der deutschen Kalkulation entspricht, minutenweise abgerechnet:

- Chirurg mit 175 EUR

- Anästhesist mit 105 EUR

- Postoperative Überwachung mit 40 EUR

Sachkosten über 20 EUR müssen vom Operateur zusätzlich bezahlt werden.

\section{Vergütung nach UV-GOÄ}

\section{Beispiel Metallentfernung kleine Röhrenknochen}

Wie aus $\bullet$ Tab. 3 ersichtlich, vergütet die BG Metallentfernungen an kleinen Röhrenknochen, insbesondere Metakarpalia oder Metatarsalia, mit 15,89 EUR. Die postoperative Überwachung, bei Fällen des Autors durch eine Anästhesieschwestern durchgeführt, wird immerhin mit 41,41 EUR vergütet. Ambulante Zuschläge für Operation und Narkose gibt es keine. Zum Vergleich ist in $\mathbf{0}$ Tab. 4 die Vergütung nach EBM für einen vergleichbaren Eingriff dargestellt.

Die betriebswirtschafltiche Kalkulation der KBV auf einem Punktwert von 5,11 Cent ergibt 309,67 EUR (• Tab. 4). Die Rechnung, die der Operateur vom Operationszentrum für die Inanspruchnahme der Infrastruktur erhält, beläuft sich bei einer Operationszeit von 40 min auf 156,64 EUR - für die Operationssaalbenutzung 116,64 EUR und für die postoperative Überwachung pauschal 40 EUR. Vergütet von der BG werden aber nur 8o,54 EUR (• Tab. 3)! 


\begin{tabular}{|llll}
\hline Tab. 1 & Beispiel für Sachkosten und Vergütung bei ambulanten Operationen \\
\hline Kostenart & Kosten [EUR] & Vergütung [EUR] & Nicht gedeckter Anteil [\%] \\
\hline 200 (Verband) & 1,36 & 1,28 & 6 \\
\hline 209 (Tape) & 21,87 & 14,94 & 32 \\
\hline $228 \mathrm{a}$ & 7,67 & 5,33 & 31 \\
\hline $237 \mathrm{~b}$ & 37,21 & 29,10 & 22 \\
\hline 2001 & 5,47 & 5,41 & 1 \\
\hline 2004 & 9,46 & 9,40 & \\
\hline
\end{tabular}

Tab. 2 Einkaufspreise für bei einem Kleinsteingriff benötigtes Material

\begin{tabular}{|ll}
\hline Material & Einkaufpreis [EUR] \\
2 Operationsmäntel & 7,98 \\
\hline 2 Operationshauben & 0,40 \\
\hline 2 Masken & 0,23 \\
\hline Abdecktuch OP-Tisch & 1,35 \\
\hline Schlitztuchset & 7,98 \\
\hline 2 Operationshandschuhe (steril) & 0,92 \\
\hline Einmalklinge & 0,32 \\
\hline Kompressen 20 Stück & 0,12 \\
\hline Hautfaden & 6,50 \\
\hline Gesamt & $\mathbf{2 5 , 8 0}$ \\
\hline
\end{tabular}

Tab. 3 Metallentfernung kleiner Röhrenknochen, Abrechnung nach BG-GOÄ

\begin{tabular}{lll}
\hline Abrechnungsposten & Verschlüsselung & Vergütung [EUR] \\
Blutleere & 2029 & 4,29 \\
\hline Metallentfernung & 2353 & 15,89 \\
\hline Sachkosten & & 7,78 \\
\hline $\begin{array}{l}\text { Postoperative Überwachung } \\
\text { Ärztlicher Assistent } \\
\text { (Operationszeit 25 min) }\end{array}$ & 448 & 41,41 \\
\hline Gesamt & $61 a$ & 11,17 \\
\hline $\begin{array}{l}\text { Vom Operationszentrum in Rechnung gestellte Kosten } \\
\text { OP-Benutzung, 40 min }\end{array}$ & $\mathbf{8 0 , 5 4}$ \\
\hline Postoperative Überwachung & 116,64 \\
\hline Gesamt & 40,00 \\
\hline
\end{tabular}

Tab. 4 Metallentfernung Mittelhand, OPS-Prozess 5.787.35 31132, Abrechnung nach EBM

\begin{tabular}{|lll}
\hline Kostenart & Punkte & Vergütung [EUR] $^{\mathrm{a}}$ \\
\hline Operation 31132 & 4660 & 238,13 \\
\hline Postoperative Überwachung & 1400 & 71,54 \\
\hline $\begin{array}{l}\text { Gesamt } \\
\text { aBerechnet mit einer Punktepauschale von 5,11 Cent }\end{array}$ & 309,67 \\
\hline
\end{tabular}

\section{Beispiel Metallentfernung große Röhrenknochen}

In $\bullet$ Tab. 5 und 6 sind die entsprechenden Werte für die Metallentfernung an großen Röhrenknochen dargestellt.

Aus - Tab. 5 ist ersichtlich, dass die gesamte ärztliche Leistung mit 312,57 EUR vergütet wird - nach Abzug der Sachkosten. Bei einer Abrechnung nach GKVSystem würde man dagegen für den gleichen Eingriff 127 EUR mehr erhalten (• Tab.6). Die Minimalausstattung der Kosten beträgt, wie oben ausgeführt (- Tab. 2) 25,80 EUR. Sie ist aber für Eingriffe bei einem großen Röhrenknochen bei weitem nicht ausreichend. Die Rechnung vom Operationszentrum würde sich für diesen Eingriff auf 258,79 EUR belaufen, hinzukommen noch die Sachkosten, die den Betrag von 20 EUR überschreiten, schätzungsweise etwa 30-40 EUR.
Nach Abzug aller Kosten verbleiben für $1 \mathrm{~h}$ Operation etwa 20 EUR, von denen der Operateur seine Mitarbeiter und die Kosten in der Praxis bezahlen muss abgesehen von seinen persönlichen Entnahmen.

\section{Beispiel eines kleinen Eingriffs}

Wie aus Tab. 7 ersichtlich, erhält der Autor für eine große Wundversorgung Sachkosten von 11,99 EUR vergütet, die tatsächlich anfallenden Sachkosten betragen aber 18,6o EUR.

\section{Kostenarten}

Weitere Beispiele für die unzureichende Vergütung nach UV-GOÄ sind die vollkommen überholten operativen Verfahren, anhand derer abgerechnet werden kann, wie sich am Beispiel der Osteosynthesen zeigt:

- Für 2347, Nagelung und/oder Drahtung eines gebrochenen kleinen Röhrenknochens, werden 32,78 EUR und 27,60 EUR (Zuschlag 442) vergütet.

- Für 2349, Nagelung und/oder Verschraubung (mit Metallplatten) eines großen Röhrenknochens, werden 95,35 EUR und 89,73 EUR (Zuschlag 444) bezahlt.

- Arthroskopien an Schultergelenk, Handgelenk oder OSG existieren in der UV-GOÄ nicht!

Diese Beträge zeigt deutlich, wie unzureichend die Vergütung durch die UV-GOÄ ist. Diese Problematik zieht sich durch die gesamte UV GOÄ hindurch:

- Es erfolgt keine ausreichende Berücksichtigung der vorzuhaltenden OPInfrastruktur.

- Operationszuschläge spiegeln nicht den tatsächlichen Kostenaufwand wider.

- Qualitätsanforderungen, die in den letzten Jahren massiv gestiegen sind und immer teurere Investitionen erfordern, finden sich in der Vergütung nicht wieder.

- Hygienerichtlinien, die beachtet werden müssen, finden sich in der Vergütung nicht wieder. 
Tab. 5 Metallentfernung große Röhrenknochen, Abrechnung nach BG-GOÄ

\begin{tabular}{lll}
\hline Abrechnungsposten & Verschlüsselung & Vergütung [EUR] \\
Blutleere & 2029 & 4,29 \\
\hline Metallentfernung & 2354 & 79,37 \\
\hline Sachkosten & & 23,33 \\
\hline Zusatz ambulante OP & 445 & 151,85 \\
\hline Postoperative Überwachung & 448 & 41,41 \\
\hline $\begin{array}{l}\text { Ärztlicher Assistent } \\
\text { (Operationszeit 57 min) 2-fach }\end{array}$ & 61 a & 22,34 \\
\hline Kompressionsverband & $203 a$ & 8,16 \\
\hline Sachkosten & & 3,88 \\
\hline Redon-Dränage & 2015 & 5,15 \\
\hline Sachkosten & & - \\
\hline Gesamt & & 339,78 \\
\hline Abzüglich Sachkosten & 27,21 \\
\hline Ärztliche Leistung & 312,57 \\
\hline Vom Operationszentrum in Rechnung gestellte Kosten & \\
\hline OP-Benutzung, 75 min & 218,70 \\
\hline $\begin{array}{l}\text { Postoperative Überwachung } \\
\text { Gesamt } \\
\text { aln diesem Betrag sind die Sachkosten noch nicht enthalten }\end{array}$ & 40,00 \\
\hline
\end{tabular}

Tab. 6 Metallentfernung großer Röhrenknochen, OPS-Prozess 5.787.35, Abrechnung nach EBM

\begin{tabular}{|c|c|c|c|}
\hline Kostenart & Verschlüsselung & Punkte & Vergütung [EUR] ${ }^{\mathrm{a}}$ \\
\hline Operation & 31132 & 6570 & 335,73 \\
\hline $\begin{array}{l}\text { Postoperative } \\
\text { Überwachung }\end{array}$ & 31504 & 2030 & 103,73 \\
\hline Sachkosten & & & Über Sprechstundenbedarf \\
\hline Gesamt & & 8600 & 439,46 \\
\hline
\end{tabular}

\section{Tab. 7 Große Wundversorgung}

\begin{tabular}{|c|c|c|c|}
\hline \multirow[t]{2}{*}{ Kostenart } & Vergütung & \multirow[t]{2}{*}{ Material } & Anfallende Kosten \\
\hline & [EUR] & & [EUR] \\
\hline 2004 & 20,62 & Schlitztuchset & 7,98 \\
\hline Sachkosten & 9,40 & Einmalskalpell & 0,61 \\
\hline 491 & 10,39 & Hautfaden & 6,50 \\
\hline \multirow[t]{2}{*}{ Sachkosten } & 2,59 & Einmalhandschuh & 0,92 \\
\hline & & LA-(Material) & 2,59 \\
\hline Gesamt & 43,00 & & \\
\hline Davon Sachkosten & 11,99 & Gesamt & 18,60 \\
\hline
\end{tabular}

- Die Dokumentation und die überbordende Bürokratie finden sich in der Vergütung nicht wieder.

\section{Lösungsvorschläge}

Zur Abrechnung können verwendet werden:

- In der UV-GOÄ aufgeführte Pauschalen
Trauma Berufskrankh 2008 10

[Suppl 1]:95-98

DOI 10.1007/s10039-007-1249-9

C) Springer Medizin Verlag 2007

\section{Haack}

\section{Kommt der Durchgangsarzt auf seine Kosten?}

\section{Zusammenfassung}

Die deutschen Durchgangsärzte arbeiten mit einer sowohl medizinisch als auch finanziell seit langem überholten Gebührenordnung. Im vorliegenden Vortrag wird insbesondere Bezug auf ambulant durchgeführte Operationen genommen. Diese werden mit der gesetzlichen Gebührenordnung in Bezug gesetzt, die nach betriebswirtschaftlichen Aspekten mit einem Punktwert von 5,11 Cent kalkuliert ist. In allen Bereichen schneidet die UV-GOÄ schlechter ab als der EBM 2005. Auch unter dem Aspekt einer Mischkalkulation rechnet sich in weiten Bereichen das ambulante Operieren im Rahmen der UV-GOÄ nicht.

\section{Schlüsselwörter}

Durchgangsarzt · Gebührenordnung ·

Ambulante Operation - Vergütung .

Wirtschaftlichkeit

\section{Are doctors on emergency call-out covering their costs?}

\section{Abstract}

German emergency doctors are working to a payment system that has long been obsolete from both the medical and the financial viewpoint. In this address, particular attention is paid to operations performed as day surgery. These are compared with the legally laid down system of remuneration, which is calculated according to economic aspects with a rate of 5.11 cents/point. In all sectors, fees calculated according to UV-GOÄ (Unfallversicherung-Gebührenordnung für Ärzte [accident insurance code of fees for doctors of medicine]) turn out less favourably than when EBM (Einheitlicher Bewertungsmaßstab [valuation criterion] 2005) is applied. When a mixed calculation is used there are still quite large sectors in which day surgery is not financially worthwhile.

\section{Keywords}

Emergency doctor on call · Code of fees - Day surgery - Remuneration · Economic efficiency 


\section{Resümee}

Die massiven Defizit der UV-GOÄ sind:

- Keine Anpassung an die Entwicklung in der modernen Chirurgie, ASK usw.

- Unzureichende Darstellung des Leistungsgeschehens in der Chirurgie

- Unzureichende Anpassung der ärztlichen und Sachkosten an die tatsächlichen Kosten

- Massive Unterdeckung der ärztlichen und technischen Vergütung

\section{Fazit}

Die Vorteile der ambulanten Operation aus betriebswirtschaftlicher und ärztlicher Sicht sind:

- kurze Wechselzeiten

- niedrige Kostenstrukturen

- wechselnde 24-h-Rufbereitschaft

Die Ertrags- und Kostensituation sind derzeit ungenügend berücksichtigt, was einer Verbesserung bedarf. Da aber der Kosten- und Ertragsfaktor bei der monistischen Bezahlung im ambulanten Bereich eine entscheidende Rolle spielen, kann unter diesem Aspekt nicht jede ambulant mögliche Operation auch ambulant durchgeführt werden.

Somit bestehen autorenseits folgende Forderungen:

- Wir fordern eine der arzt- und technischen Leistung gerechte Vergütung unserer konservativen und operativen Tätigkeit.

- Mein Angebot an die Versicherungsträger steht: Reden Sie mit dem BV der Niedergelassenen Chirurgen (BNC), da wir die aktuellen Daten verfügbar haben.

\section{Korrespondenzadresse}

\section{Dr. D. Haack}

Berufsverband Niedergelassener Chirurgen, Wulfsdorfer Weg 7, 22359 Hamburg

Haack-kerber@t-online.de

Interessenkonflikt. Der korrespondierende Autor gibt an, dass kein Interessenkonflikt besteht. 